Research Paper

\title{
Huaier Restrains Proliferative and Migratory Potential of Hepatocellular Carcinoma Cells Partially Through Decreased Yes-Associated Protein 1
}

\author{
Liang Shan ${ }^{1 *}$, Yan $\mathrm{Li}^{2 *}$, Hongyuan Jiang1, Yuquan Tao1, Zijun Qian ${ }^{1}$, Lan $\mathrm{Li}^{3}$, Feng Cai ${ }^{3}$, Lifang Ma ${ }^{\circledR}$, \\ Yongchun $\mathrm{Yu}^{1 凶}$ \\ 1. Shanghai Municipal Hospital of Traditional Chinese Medicine, Shanghai University of Traditional Chinese Medicine, Shanghai, 200071, P.R. China; \\ 2. Department of Oncology, Shanghai Municipal Hospital of Traditional Chinese Medicine, Shanghai University of Traditional Chinese Medicine, Shanghai, \\ 200071, P.R. China; \\ 3. Department of Clinical Laboratory, Shanghai Municipal Hospital of Traditional Chinese Medicine, Shanghai University of Traditional Chinese Medicine, \\ Shanghai, 200071, P.R. China. \\ * These two authors contributed equally to this study.
}

$\triangle$ Corresponding authors: Dr. Lifang Ma, No.274 Middle Zhijiang Road, Jingan District, Shanghai, P.R. China, 200071 Fax and Tel: 86-21-56634252 E-mail: malifang0606118@126.com Prof. Yongchun Yu, No.274 Middle Zhijiang Road, Jingan District, Shanghai, P.R. China, 200071 Fax and Tel: 86-21-66313245 E-mail: yuyongchun1255@126.com

(c) Ivyspring International Publisher. This is an open access article distributed under the terms of the Creative Commons Attribution (CC BY-NC) license (https://creativecommons.org/licenses/by-nc/4.0/). See http://ivyspring.com/terms for full terms and conditions.

Received: 2017.05.15; Accepted: 2017.10.13; Published: 2017.11.06

\begin{abstract}
In China, Trametes robiniophila Murr (Huaier), a traditional Chinese herbal medicine, has been widely used in adjuvant therapies of hepatocellular carcinoma (HCC). However, the molecular mechanisms have not been fully understood. The aims of this study are to investigate the functions and mechanisms of Huaier on inhibiting proliferation and migration of HCC cells. Firstly, cell counting kit-8 (CCK-8) and colony formation shown Huaier inhibited proliferation of HCC Bel-7404, Bel-7402 and SMMC-7721 cells in a dose-dependent manner, and this inhibition might be due to Huaier decreased the expressions of the proliferating cell nuclear antigen (PCNA), the nuclear proliferation related antigen (Ki-67) and CyclinDI detected by western blotting analysis. Notably, we also found Huaier treatment did not cause any cytotoxicity to normal human hepatocyte L-02 cells. Next, we found Huaier dose-dependently decreased Bcl-2 expression and increased Bax expression in Bel-7404 cells. The activities of cleaved caspase substrates had also been enhanced after Huaier treatment, suggesting Huaier treatment could induce HCC cell apoptosis. Then, the inhibitory effects of Huaier on migration of Bel-7404, Bel-7402 and SMMC-7721 cells via inhibiting Epithelial mesenchymal transition (EMT) had also been proved. Moreover, we confirmed yes-associated protein 1 (YAPI) was up-regulated in HCC cells and tissues, and overexpression of YAPI promoted HCC cell proliferation and migration. Then, western blot and immunefluorescence shown Huaier had the inhibitory effects on YAPI in HCC cells. On the other hand, human liver cancer tissue microarray (TMA) shown YAPI expression was closely to clinic. Our study also confirmed Huaier had the inhibitory effects on YAPI in xenograft mice models, it could be because Huaier treatment translocated YAPI from nucleus to cytoplasm, and further promoted phosphorylation of YAPI to be degraded by ubiquitination. Hence, we conclude that Huaier may restrain the proliferation and migration of HCC cells via down-regulation of YAPI. In summary, our study reveals the potential mechanisms of Huaier on inhibiting proliferation and migration of HCC cells. Importantly, for the first time, we found that Huaier can inhibit YAPI expression in this anti-tumor process. We believe this finding is beneficial for the clinical applications of Huaier and the targeted therapies for HCC.
\end{abstract}

Key words: Huaier, hepatocellular carcinoma, YAP, Hippo, targeted treatment. 


\section{Introduction}

Liver cancer, especially HCC, is a common malignant tumor in clinic and one of the leading causes of cancer death worldwide [1]. At present, the treatment methods for HCC are mainly surgery, radiotherapy and chemotherapy, however, the recovery rate is extremely low. For the above reasons, Traditional Chinese Medicine (TCM) is widely used in adjuvant therapies of $\mathrm{HCC}$ in China. Huaier, a traditional Chinese herbal medicine, has been confirmed the successful anti-tumor effects in clinical trials. In addition, some studies shown that Huaier polysaccharides $(\mathrm{HP})$, the main active ingredients in this herb, were able to induce HCC cell apoptosis, inhibit angiogenesis and enhance immunity [2-4]. However, notably, the inhibitory effects of HP on HCC cells are not as good as the traditional herbal medicine. Hence, an increasing number of studies focus on the Huaier aqueous extract. Recently, correlative studies have made considerable progress, however, the possible targets of Huaier in this anti-tumor process still keep unclear, which limits the potential for drug development.

In this study, we explored the inhibitory effects of Huaier on proliferation and migration of HCC cells. To clarify the potential mechanisms of Huaier treatment, we tested the expression levels of PCNA, $\mathrm{Ki}-67, \mathrm{CyclinD1}$, E-cadherin and $\mathrm{N}$-cadherin in Bel-7404, Bel-7402 and SMMC-7721 cells. We also measured the expressions of Bcl-2, Bax and cleaved caspase substrates, the apoptosis markers of HCC cells, after Huaier treatment. Additionally, CCK-8 and colony formation shown Huaier treatment did not cause any cytotoxicity to normal human hepatocyte L-02 cells. We then tested the expression levels of E-cadherin and N-cadherin in L-02 cells after Huaier treatment, indicating a very favorable sensitive profile of Huaier to HCC cells.

Remarkably, hepatocarcinogenesis is regulated by complicated factors, and Huaier has multi-targets in the anti-tumor process. Numerous studies have reported that Hippo signaling pathway is a key for HCC cell proliferation and transformation, and YAP1 is the core transcription activator in this signal [5]. Because YAP1 plays a critical role in hepatocarcinogenesis, we hypothesize that Huaier may also restrain HCC cells via down-regulating YAP1.

\section{Materials and Methods}

\section{Cell culture and vectors}

L-02, Bel-7404, Bel-7402, SMMC-7721 and HepG2 cell lines were cultured in DMEM (Hyclone, Beijing,
China) with $10 \%$ fetal bovine serum and $1 \%$ penicillin-streptomycin at a $37^{\circ} \mathrm{C}$ cell culture incubator with $5 \% \quad \mathrm{CO}_{2}$. Complementary DNA fragments encoding human YAP1 was cloned into PGIPZ-based lentiviral vector. The primers are listed in Table 1.

Table 1. The clonal primers for YAP5SA.

\begin{tabular}{ll}
\hline Name & Sequences $\left(5^{\prime}-3^{\prime}\right)$ \\
\hline YAP5SA-Flag-F & $\begin{array}{l}\text { ctaggctagcgattacaaggatgacgatgacaaggattacaag } \\
\text { gatgacgatgacaaggattacaaggatgacgatgacaagctt } \\
\text { catggcggcegcttactataaccatgtaagaaagcttctttatc }\end{array}$ \\
YAP5SA-R
\end{tabular}

\section{Huaier}

The granules of Huaier was obtained from Qidong Gaitianli Medicine Co. Ltd (Jiangsu, China). The drug was dissolved with enzyme-free water to a final concentration of $300 \mathrm{mg} / \mathrm{ml}$. Then, collecting supernatant after centrifugation at $1000 \mathrm{rpm}$ for $1 \mathrm{~min}$. Storing at $4^{\circ} \mathrm{C}$ for short-term use.

\section{Measurements of cell viabilities by CCK-8 and colony formation}

\section{CCK-8}

15000 cells were inoculated and treated with Huaier $(0,5,10$ and $15 \mathrm{mg} / \mathrm{ml})$ for $24 \mathrm{~h}$ in 96-well plates. Then, the old medium was replaced with 10ul CCK-8 and 100ul fresh medium, and cells were incubated for $1 \mathrm{~h}$ at $37^{\circ} \mathrm{C}$ until the color turned orange. The optical density (OD) at $450 \mathrm{~nm}$ was read using a microplate reader.

\section{Colony formation}

500 cells were seeded in 6-well plates for colony formation, after $4 \mathrm{~h}$, cells were treated with Huaier $(0$ and $15 \mathrm{mg} / \mathrm{ml}$ ) at $37^{\circ} \mathrm{C}$ in an incubator, after 7 days, colonies were washed twice with PBS (pH7.4) and fixed with $95 \%$ ethanol. Then, cells were stained with $0.1 \%$ crystal violet for $10 \mathrm{~min}$ after air drying. Taking a picture of cell colonies.

\section{Measurements of cell migration activities by wound healing assay and transwell assay}

\section{Wound healing assay}

Cells were seeded in 6-well plates for wound healing assay, after cell attachment, gently scratching a straight line on the cell layer with a $200 \mu 1$ yellow pipet tips, and then washing cells with PBS (pH7.4) for 3 times, after that, adding $2 \mathrm{ml}$ complete medium containing different concentrations of Huaier $(0$ and $15 \mathrm{mg} / \mathrm{ml}$ ). Selecting the same point of view to take a picture at $0 \mathrm{~h}, 12 \mathrm{~h}$ and $24 \mathrm{~h}$. The cell scratch area was measured by ImageJ $1.74 \mathrm{v}$. 


\section{Transwell assay}

Putting transwell chambers into a 24-well plate. $200 \mu 1 \mathrm{DMEM} /$ chamber with $5 \times 10^{4}$ cells was added to the upper chamber. $500 \mu \mathrm{l}$ complete medium containing Huaier $(0$ and $15 \mathrm{mg} / \mathrm{ml}$ ) was added to the 24-well plate, and then cells were incubated at a $37^{\circ} \mathrm{C}$ incubator. After $24 \mathrm{~h}$, cells on the lower surface of transwell membranes were washed twice with PBS (pH7.4) and fixed with 95\% ethanol for 10min. Next, cells were stained with $0.1 \%$ crystal violet for $10 \mathrm{~min}$ after air drying. Taking a picture of cells stained.

\section{Quantitative RT- PCR}

Total RNA was extracted using TRIzol Reagent, and cDNA was synthetized with All-in-One cDNA Synthesis SuperMix (biotool, B24403), then, qPCR was performed using $2 \times$ SYBR Green qPCR Master Mix (biotool, B21202). The specific primers are listed in Table 2.

Table 2. Primers used for qRT-PCR.

\begin{tabular}{|c|c|}
\hline Name & Sequences $\left(5^{\prime}-3^{\prime}\right)$ \\
\hline GAPDH-F & ATCATCCCTGCCTCTACTGG \\
\hline GAPDH-R & GTCAGGTCCACCACTGACAC \\
\hline YAP1-F & CCTCGTTTTGCCATGAACCA \\
\hline YAP1-R & GTTCTTGCTGTTTCAGCCGC \\
\hline Lats1-F & GACTTGCAAGCTGCTGGATT \\
\hline Lats1-R & AGGATTCTTTAGAACCTTTC \\
\hline Lats2-F & СТСАСААТTССАААТАТTAС \\
\hline Lats2-R & GCGTGTTCTCCCAGTTGATC \\
\hline CTGF-F & TGCCCTCGCGGCTTACCGAC \\
\hline CTGF-R & TGCAGGAGGCGTTGTCATTG \\
\hline$\beta$-Catenin-F & CATCTACACAGTTTGATGCTGCT \\
\hline$\beta$-Catenin- $R$ & GCAGTTTTGTCAGTTCAGGGA \\
\hline GSK3 $\beta-F$ & АССТCTGGCTACСАТССТTATTCС \\
\hline GSK3 $\beta-R$ & GTCCACGGTCTCCAGTATTAGCA \\
\hline APC-F & CAGATTGATTTATTAGAGCGTC \\
\hline APC-R & TGAACCCATAGGAACAGG \\
\hline AXIN2-F & CTGAAGCCTGCCACCAAGA \\
\hline AXIN2-R & TAGGCATTTTCCTCCATCACC \\
\hline TCF4-F & ATGTTTTCACCTCCTGTGAG \\
\hline TCF4-R & GACAAAAGGTGGAGAGAGAT \\
\hline SOX9-F & GCACTCATAATATGGCATCCTTCA \\
\hline SOX9-R & AAACACGAACACAAACCAAAGCTT \\
\hline SMAD7-F & CCTTAGCCGACTCTGCGAAC \\
\hline SMAD7-R & TGCCACCACGCACCAGTGTG \\
\hline TGF $\beta R 1-F$ & ACGGCGTTACAGTGTTTCTG \\
\hline TGF $\beta R 1-R$ & GCACATACAAACGGCCTATCTC \\
\hline
\end{tabular}

\section{Western blotting analysis}

Cells were treated with a series concentrations of Huaier $(0,5,10$ and $15 \mathrm{mg} / \mathrm{ml})$ for $24 \mathrm{~h}$, and then lysed by cell lysis buffer for Western and IP (Beyotime, P0013). Protein concentration was detected with BCA Protein Assay Kit (KeyGen BioTECH, KGP902). After that, $20 \mu \mathrm{g}$ proteins were separated by $10 \%$ or $12 \%$ SDS-PAGE and transferred onto Pure Nitrocellulose Blotting Membrane (Pall Corporation, P/N 66485). Then, the blots were blocked for nonspecific binding with 5\% non-fat milk in PBST (PBS, Tween-20, pH7.4) at room temperature for $1 \mathrm{~h}$. Next, the blots were incubated overnight at $4^{\circ} \mathrm{C}$ with $5 \%$ non-fat milk containing primary antibodies which are listed as follows: anti-PCNA (ImmunoWay, YM3031), anti-Ki-67 (ImmunoWay, YT2467), anti- $\beta$-actin (ImmunoWay, YM3028), anti-Bcl-2 (ImmunoWay, YM3041), anti-Bax (ImmunoWay, YT0455), anti-E-cadherin (ImmunoWay, YT1454), anti-N-cadherin (ImmunoWay, YT2988), anti-YAP1 (abcam, ab52771), anti-p-YAP1 (ab76252), anti-CyclinD1 (abcam, ab134175), anti-Cleaved Caspase Substrate Motif (Cell Signaling, \#8698) and anti-YAP/TAZ (Cell Signaling, \#8418). After that, incubating blots with secondary antibodies conjugated with HRP (Cell Signaling, \#7074 or \#7076) for $1 \mathrm{~h}$ at room temperature. Finally, after washing three times with PBST, the blots were visualized by New Super ECL Assay (KeyGen BioTECH, KGP1128).

\section{Human liver cancer tissue microarray (TMA)}

The TMA slides were purchased from U.S. Biomax (Rockville, MD). The slides were incubated with anti-YAP1 (abcam, ab52771).

\section{Immunofluorescence}

For IF, cells were cultured on cover slips and treated with Huaier $(0$ and $15 \mathrm{mg} / \mathrm{ml})$ for $24 \mathrm{~h}$. Cells were fixed with $200 \mu 14 \%$ Paraformaldehyde (PFA) for $15 \mathrm{~min}$, and then washed with PBS (pH7.4) for 3 times, each time for 5 minutes. Next, $200 \mu$ l blocking buffer consisting of 3\% FBS, $1 \%$ Goat-serum, $0.1 \%$ Triton $\mathrm{X}-100$ was added to each well for $1.5 \mathrm{~h}$ at room temperature. After that, cells were incubated with the primary antibody (anti-YAP1, abcam, ab52771) that diluted in blocking buffer at $4^{\circ} \mathrm{C}$ overnight before incubated with Cy3 conjugated Goat Anti-Rabbit IgG $(\mathrm{H}+\mathrm{L})$ (Wuhan Google Biotechnology, GB21303) for $1 \mathrm{~h}$ at room temperature. Cells were then incubated with DAPI for $10 \mathrm{~min}$ at room temperature. At last, observed under fluorescence microscope.

\section{Immunohistochemistry}

To establish the nude mouse xenograft model, Bel-7404 cells $\left(1 \times 10^{7}\right.$ cells, in $0.1 \mathrm{ml}$ PBS, pH7.4) were subcutaneously injected into the left armpit of a athymic BALB/c nude mouse (SLAC, Shanghai, China). Two weeks later, after xenografts were visible, the mice were treated with Huaier (100ul of solution containing $50 \mathrm{mg}$ ) or normal saline. Then, all the mice were sacrificed after treatment for consecutive 14 days. Next, tumor xenografts were fixed in paraformaldehyde for $12 \mathrm{~h}$ and embedded in paraffin. After that, KeyGEN One-Step IHC Assay (KeyGen BioTECH: KGOS60) were used to stain 5um thickness 
of paraffin sections, and the paraffin sections were incubated with anti-YAP1 (abcam, ab52771). Taking a picture of these paraffin sections. All mouse experiments were performed according to the Interdisciplinary Principles and Guidelines for the Use of Animals in Research, Testing, and Education by the New York Academy of Sciences, Ad Hoc Animal Research Committee.

\section{Statistical analysis}

Differences between treatment group and control group were analyzed with student's t-test in IBM SPSS Statistics 21. It was considered to be statistically significant when $p<0.05$. The correlation of YAP1 protein expression with clinicopathologic features was analyzed with chi-square.

\section{Results}

\section{Huaier inhibited proliferation and induced apoptosis of HCC cells}

Bel-7404, Bel-7402 and SMMC-7721 cells were treated with Huaier, we found the inhibitory effects of Huaier on proliferation were dose-dependent (Figure 1A). Then, we performed a colony formation assay, results shown Huaier effectively inhibited the formation of colonies derived from Bel-7404, Bel-7402 and SMMC-7721 cells compared with control group (Figure 1B). On the other hand, we found Huaier treatment did not cause any cytotoxicity to L-02 cells, suggesting a very favorable sensitive profile of Huaier to HCC cells (Figure 1A-B). We have confirmed Huaier was able to inhibit Bel-7404, Bel-7402 and SMMC-7721 cell proliferation, but, what were the targets of Huaier in this process? Next, we tested three well-known markers of cell cycle activities, PCNA, Ki-67 and CyclinD1, as shown in Figure 1C, Huaier observably decreased the expression levels of PCNA, Ki-67 and CyclinD1 in Bel-7404, Bel-7402 and SMMC-7721 cells at a does-dependent manner. Together, these results indicate that Huaier may be able to inhibit HCC cell proliferation through restraining PCNA, Ki-67 and CyclinD1.

Having proved that Huaier treatment significantly inhibited the HCC cell proliferation, we next investigated the apoptosis-induced mechanisms of Huaier treatment. Bcl-2 and Bax were examined in the present experiment. As seen in Figure 1D, Bcl-2 expression was down-regulated when treated with Huaier in Bel-7404 cells, whereas Bax expression was up-regulated. The aspartic acid-directed cysteine proteases (Caspases) are central to the apoptotic mechanisms [6, 7]. Next, we tested cleaved caspase substrate levels after Huaier treatment in Bel-7404 and SMMC-7721 cells. Western blot shown that Huaier dose-dependently enhanced cleaved caspase substrate expressions (Figure 1E). In summary, these results show Huaier can promote apoptosis of HCC cells, which may be mediated by Bcl-2, Bax and Caspases.

\section{Huaier restrained migration of $\mathrm{HCC}$ cells via inhibiting Epithelial mesenchymal transition (EMT)}

The migration of HCC cells seriously affects the prognosis and survival of HCC patients [8]. Cell wound healing assay were performed to confirm the effects of Huaier on migration of Bel-7404 and SMMC-7721 cells. As shown in Figure 2A, Huaier treatment was able to reduce migratory distance of Bel-7404 and SMMC-7721 cells compared with control group. Additionally, results from transwell assay shown the number of HCC cells passed through the transwell membranes was significantly reduced by Huaier (Figure 2B). Collectively, these results reveal that Huaier can inhibit the migration capacity of HCC.

Next, we tested how Huaier inhibited HCC cell migration. EMT is critical for HCC progression, which is responsible for cell migration and invasion, and E-cadherin and N-cadherin are the markers of EMT [9]. Western blot was applied to detect the expressions of E-cadherin and $\mathrm{N}$-cadherin after Huaier treatment. As shown in Figure 2C, Huaier does-dependently promoted E-cadherin expression whereas inhibited $\mathrm{N}$-cadherin expression in Bel-7404, Bel-7402 and SMMC-7721 cells, however, the expressions of E-cadherin and N-cadherin in L-02 cells had little effects by Huaier. These experiments demonstrate that Huaier suppresses the migratory capacity of HCC via inhibiting EMT, and Huaier treatment has the advantage of high specificity.

\section{Overexpression of YAP 1 induced HCC cell proliferation and migration.}

Huaier has the role of multi-targets in anti-tumor process. As depicted in Figure 3A, some tumor-related mRNA expressions shown distinct change after Huaier treatment in Bel-7404 cells. As expected, we found YAP1 mRNA level was obviously reduced. Then, Western blot shown the expression levels of YAP1 in various cancer cells (Bel-7404, Bel-7402, SMMC-7721 and HepG2) were higher than its expression in L-02 cells (Figure 3B). These results suggested that YAP1 may be a potential entry point to reveal the mechanisms of Huaier treatment. Next, we proved the proliferative and migratory activities of Bel-7404 cells transfected with overexpression of YAP5SA plasmid were enhanced compared to control group, indicating YAP1 is able to promote HCC cell proliferation and migration (Figure 3C-D). 
A

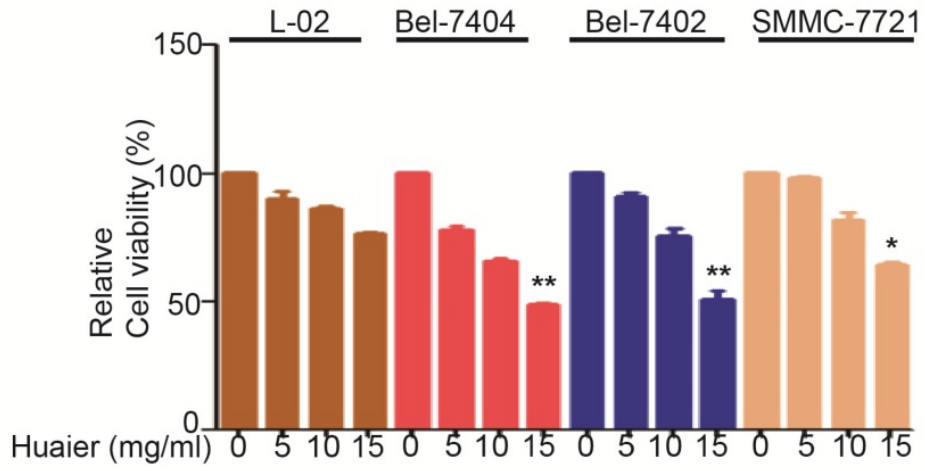

B

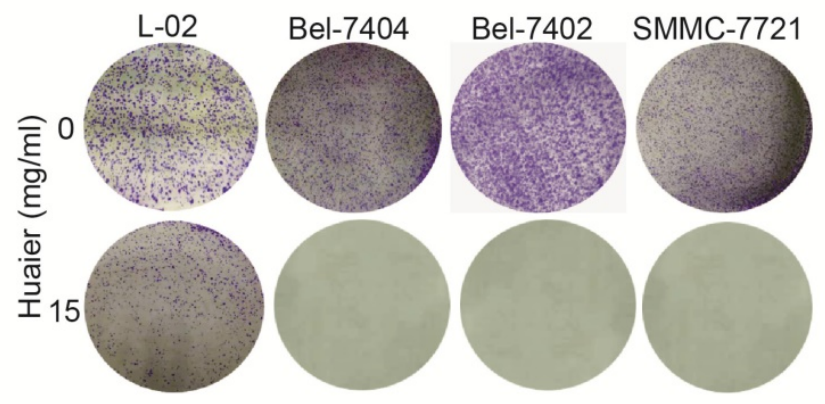

C
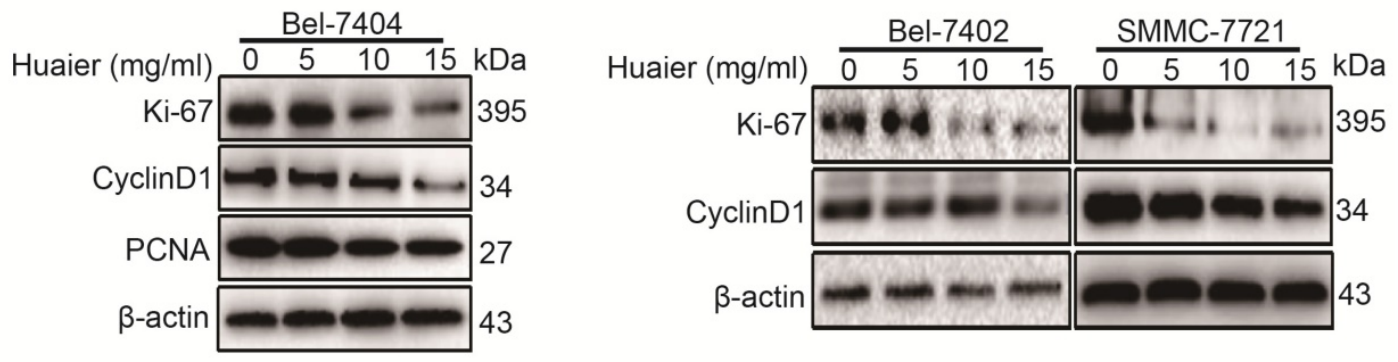

D

E
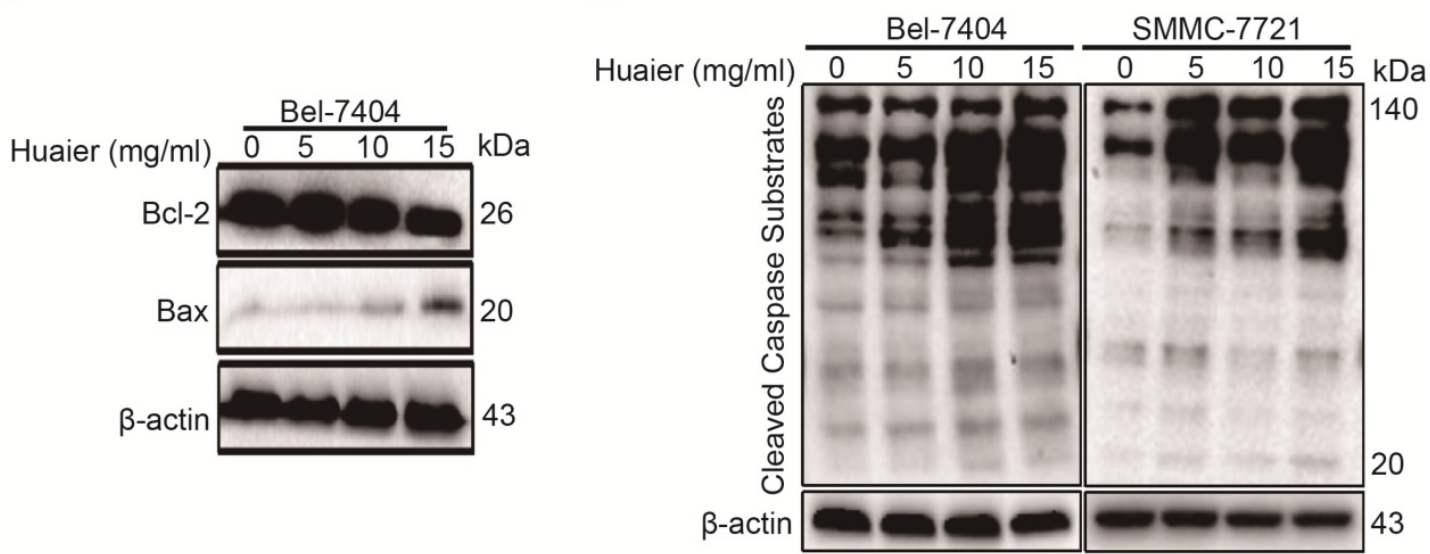

Figure 1. Huaier inhibited proliferation and induced apoptosis of HCC cells. A. Cell viabilities were measured by CCK-8 in L-02, Bel-7404, Bel-7402 and SMMC-7721 cells treated with Huaier at the concentrations of $0,5,10,15 \mathrm{mg} / \mathrm{ml}$ for $24 \mathrm{~h}$. Data was represented with GraphPad Prism 5.02 . * $<0.05$, ** $<0.01$ compared with control group. B. Colony formation was performed to test the effects of Huaier ( 0 and 15mg/ml) on L-02, Bel-7404, Bel-7402 and SMMC-7721 cells. The colonies were then stained with $0.1 \%$ crystal violet after 7 days. C. The protein expressions of Ki-67, CyclinD1 and PCNA in Bel-7404, Bel-7402 and SMMC-7721 cells treated with Huaier at the concentrations of $0,5,10,15 \mathrm{mg} / \mathrm{ml}$ for $24 \mathrm{~h}$. D. The protein expressions of Bcl-2 and Bax in Bel-7404 cells treated with Huaier at the concentrations of $0,5,10,15 \mathrm{mg} / \mathrm{ml}$ for $24 \mathrm{~h}$. E. The protein expressions of cleaved caspase substrates in Bel-7404 and SMMC-7721 cells treated with Huaier at the concentrations of $0,5,10$ and $15 \mathrm{mg} / \mathrm{ml}$ for $24 \mathrm{~h}$. 
A
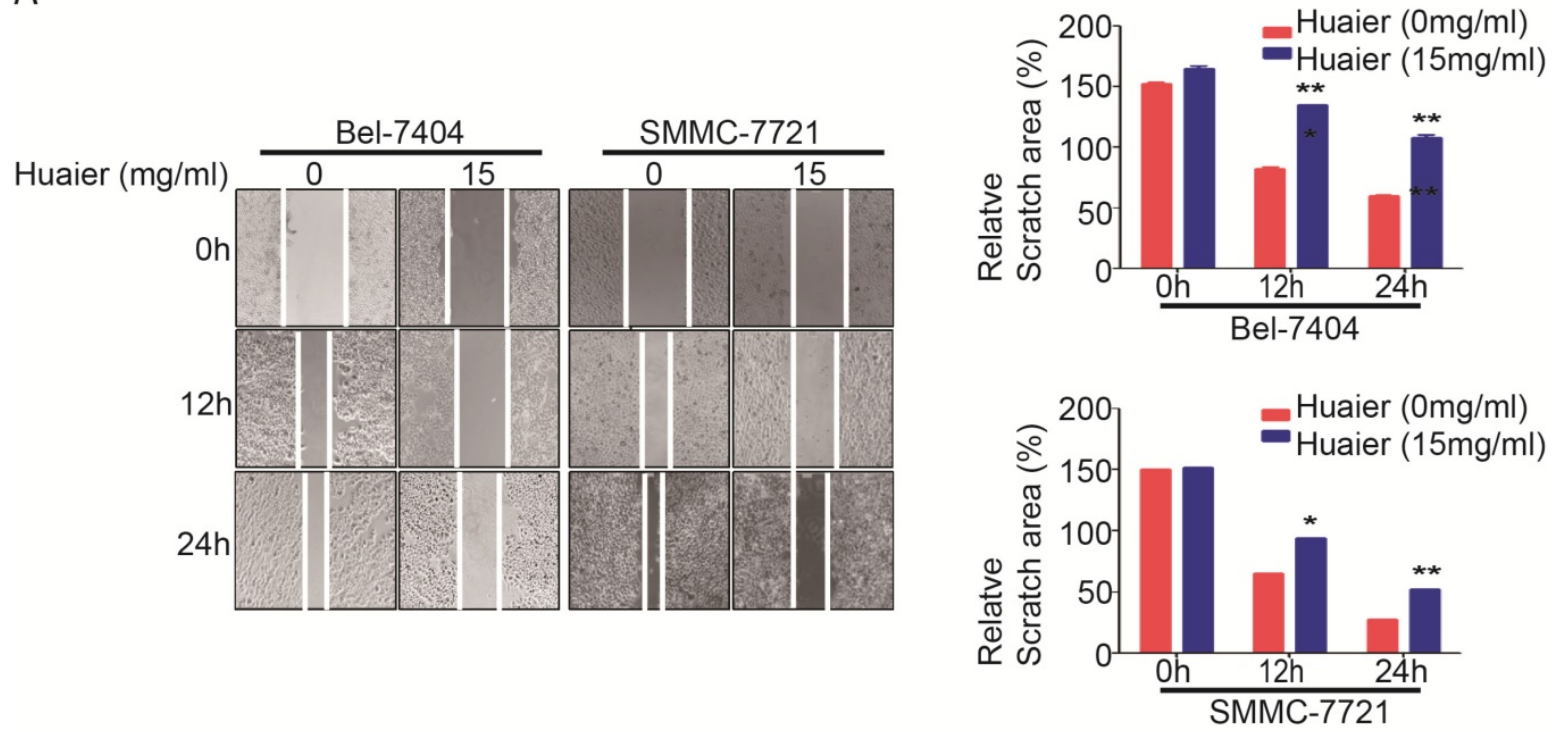

B
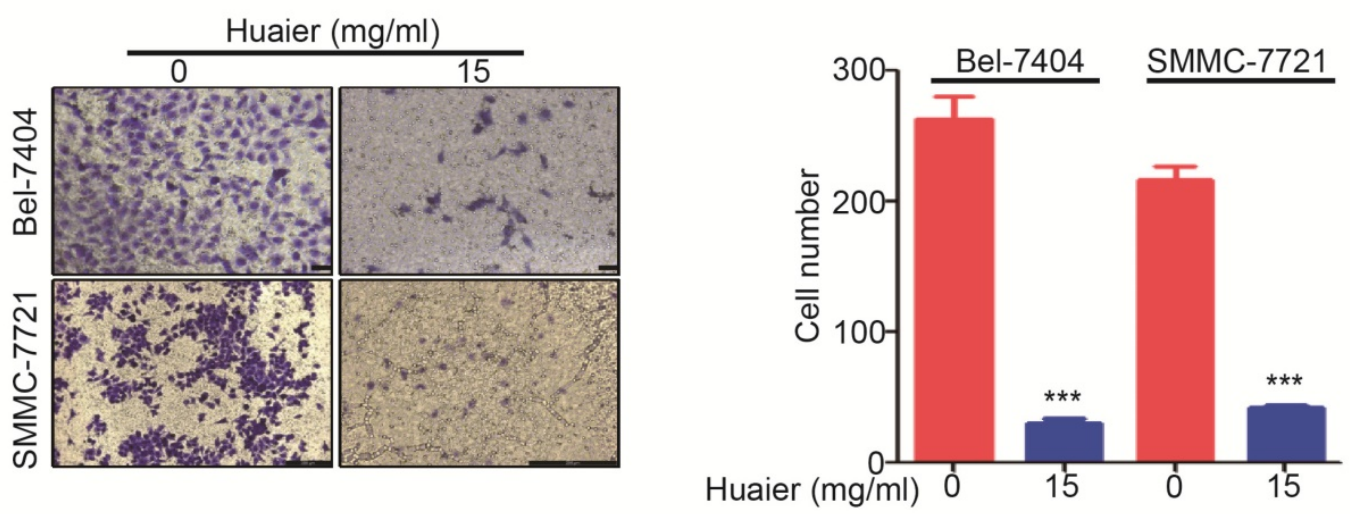

C

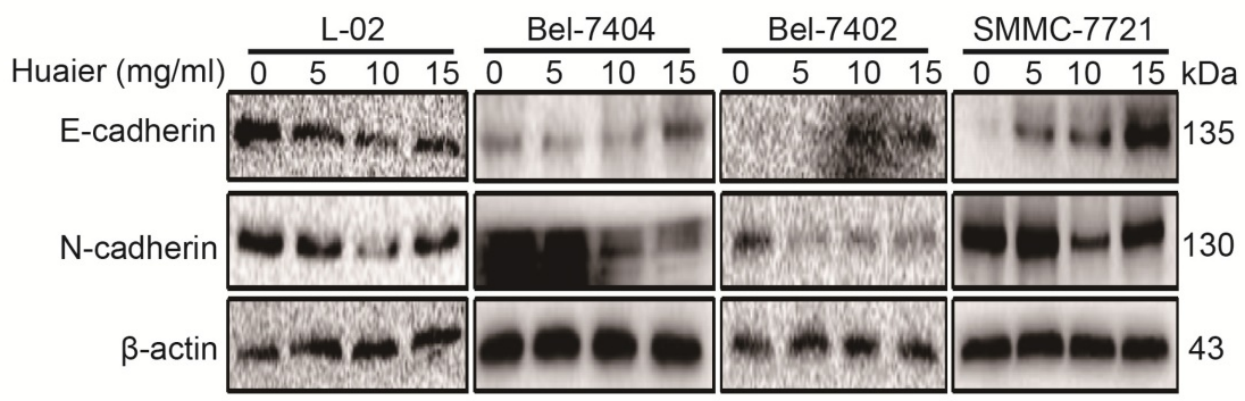

Figure 2. Huaier inhibited migration of HCC cells. A. Wound healing assay was performed to test the effects of Huaier $(15 \mathrm{mg} / \mathrm{ml})$ on Bel-7404 and SMMC-7721 cells. Selecting the same point of view to take a picture at $0 \mathrm{~h}, 12 \mathrm{~h}$ and $24 \mathrm{~h}$, and the relative scratch area was measured by Image $1.74 \mathrm{v}$. Data was represented with GraphPad Prism 5.02. ${ }^{2}<0.05,{ }^{* *} p<0.01$ compared with control group. B. Transwell assay was performed to test the effects of Huaier $(0$ and $15 \mathrm{mg} / \mathrm{ml}$, for $24 \mathrm{~h}$ ) on migration of Bel-7404 and SMMC-7721 cells. Cells on the lower surface of transwell membrane were stained with $0.1 \%$ crystal violet. Data was represented with GraphPad Prism 5.02. *** $<0.001$ compared with control group. C. The protein expressions of E-cadherin and N-cadherin in L-02, Bel-7404, Bel-7402 and SMMC-7721 cells treated with Huaier at the concentrations of $0,5,10$ and $15 \mathrm{mg} / \mathrm{ml}$ for $24 \mathrm{~h}$.

To further proved the relationship between YAP1 and clinicopathologic features of HCC, we performed IHC using TMA on greater than 120 human HCC samples. Results shown YAP1 proteins were highly expressed in most HCC patients (Figure
3E), and its expression was significantly associated with high tumor stage, tumor grade and $\mathrm{T}$ Classfication. The statistical analysis of the TMA data is shown in Table 3. 
A

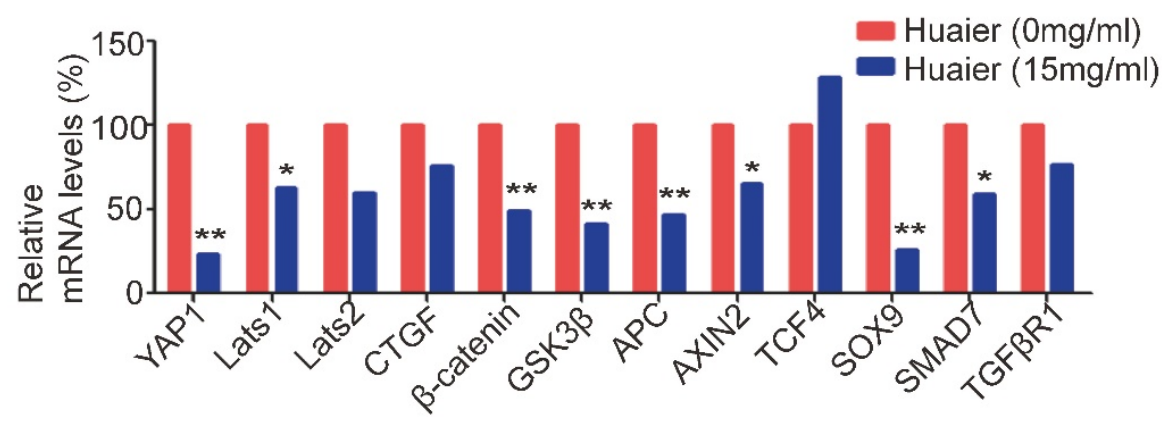

B

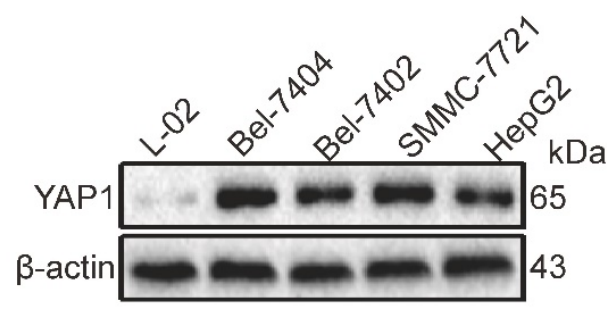

C

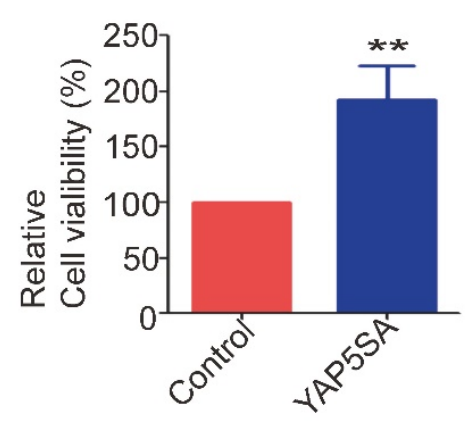

D
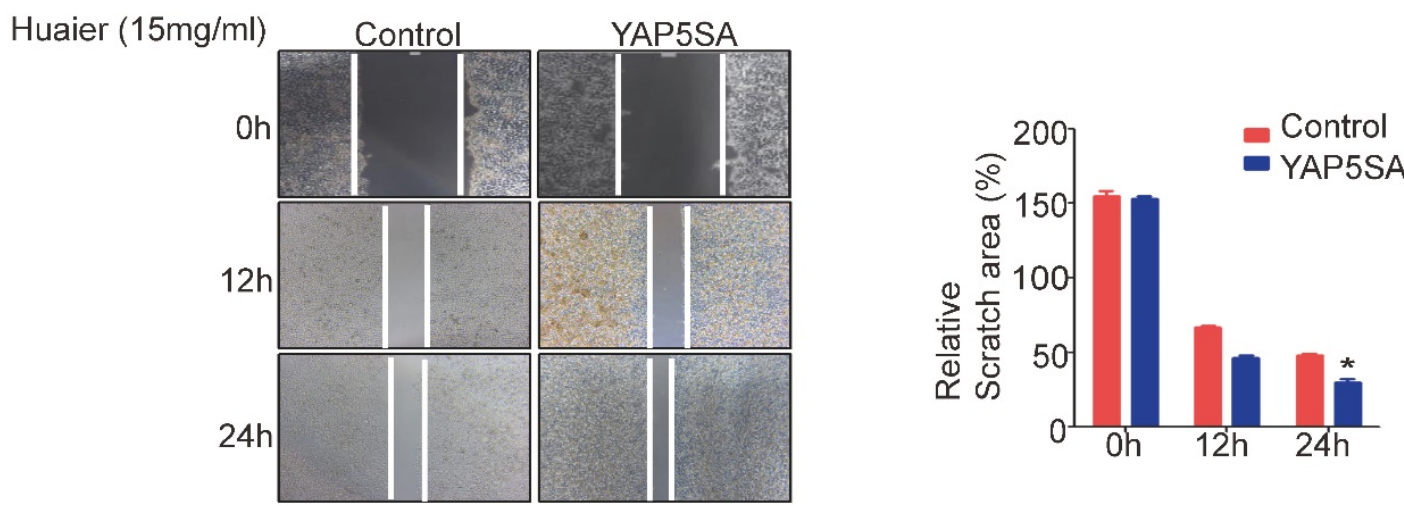

E
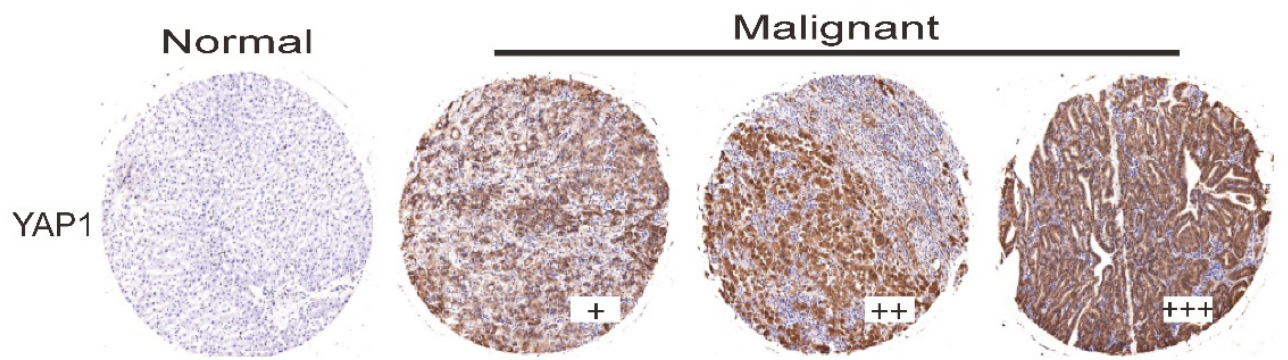

Figure 3. Overexpression of YAPI induced HCC cell proliferation and migration. A. The tumor-related mRNA expressions were measured by quantitative RT-PCR in Bel-7404 cells treated with Huaier $(15 \mathrm{mg} / \mathrm{ml})$ for $24 \mathrm{~h}$. Data was represented with GraphPad Prism 5.02 . *p $<0.05$, ${ }^{* *} \mathrm{p}<0.01$ compared with control group. B. The protein expression of YAP1 in L-02, Bel-7404, Bel-7402, SMMC-7721 and HepG2 cells. C. Cell viabilities were measured by CCK-8 in Bel-7404-control and Bel-7404-YAP5SA cells with or without Huaier treatment $(15 \mathrm{mg} / \mathrm{ml})$ for $24 \mathrm{~h}$. Data was represented with GraphPad Prism 5.02 . **p $<0.01$ compared with control group. D. Wound healing assay was performed to test the effects of YAP5SA on migration of Bel-7404 cells with or without Huaier treatment $(15 \mathrm{mg} / \mathrm{ml})$. Selecting the same point of view to take a picture at 0,12 and $24 \mathrm{~h}$, and the relative scratch area was measured by ImageJ $1.74 \mathrm{v}$. Data was represented with GraphPad Prism 5.02. * $\mathrm{p}<0.05$ compared with control group. E. Representative IHC images of YAPI staining from TMA analysis. 
Table 3. The correlation of YAPI protein expression with clinicopathologic features.

\begin{tabular}{lllll}
\hline Clinicopathologic & Cases $(\mathrm{N})$ & \multicolumn{2}{l}{ YAP1 expression } & p \\
\cline { 3 - 5 } Features & & Low & High & 0.508 \\
\hline Ages & & & & \\
$\geq 60$ & 26 & 16 & 10 & 0.117 \\
$<60$ & 94 & 51 & 43 & \\
Gender & & & & 0.000129 \\
Male & 94 & 56 & 38 & \\
Female & 26 & 11 & 15 & 0.005 \\
Grade & & & & \\
1 & 12 & 8 & 4 & \\
2 & 103 & 70 & 27 & \\
3 & 5 & 1 & 10 & \\
Stage & & & & \\
I & 6 & 5 & 1 & \\
II & 42 & 24 & 18 & \\
III & 72 & 24 & 48 & \\
T Classfication & & & & \\
T1 & 6 & 5 & 1 & \\
T2 & 42 & 24 & 18 & \\
T3 & 72 & & 51 & \\
\hline
\end{tabular}

\section{Huaier induced the down-regulation of YAP I in HCC cells}

To further reveal the mechanisms of these phenomena and search for a cure, we then tested YAP and its paralog transcriptional coactivator with PDZ-binding motif (TAZ) levels. Results shown Huaier was also able to decrease the expressions of YAP/TAZ in both Bel-7404 and SMMC-7721 cells in a does-dependent manner (Figure 4A). Because phosphorylated YAP1 was able to be degraded by ubiquitination, we also tested p-YAP1 (ser127) under Huaier treatment, and found the level of p-YAP1 (ser127) was significantly enhanced (Figure 4A-B). Next, we examined the effect of cycloheximide (CHX), a protein synthesis inhibitor, on YAP1 after Huaier intervention in Bel-7404 cells. Compared with control group, we found YAP1 begun to be degraded at $8 \mathrm{~h}$, as time goes on, YAP1 expression was already very low at $24 \mathrm{~h}$ (Figure 4C). Moreover, in xenograft mice models, we also found Huaier treatment effectively reduced YAP1 expression (Figure 4D). Collectively, these results demonstrate that Huaier is able to inhibit proliferation and migration of HCC cells in vivo and vitro, this may be via decreasing YAP1 expression at mRNA and protein levels (Figure 3A) (Figure 4A-D).

But, how Huaier induced YAP1 phosphorylation and its degradation by ubiquitination. As shown in Figure 4E, YAP1 was mainly expressed in the nucleus before Huaier treatment, as a result, YAP1 was able to exert its activity by binding to the related transcription factors. We then found Huaier treatment promoted YAP1 to exit from nucleus to cytoplasm in both Bel-7404 and SMMC-7721 cells. Relevant studies had confirmed YAP1 located in the cytoplasm would be phosphorylated by large tumor suppressor gene $1 / 2$ (Lats1/2) under the regulation of mps 1 binder kinase activator-like 1 (Mob1). Then, p-YAP1 (ser127) bound 14-3-3 and, finally, YAP1 was degraded by ubiquitination [10].

In summary, all the results indicate that YAP1 promotes HCC cell proliferation and migration and Huaier is able to inhibit YAP1 in vivo and in vitro. YAP1 may be a critical target in the anti-tumor process of Huaier treatment.

\section{Discussion}

Huaier has been used in TCM for approximately 1,600 years. Clinical applications and researches have confirmed Huaier is able to inhibit proliferation, induce apoptosis and reverse drug resistance of HCC cells [11]. However, the exact mechanisms still keep unclear, which limits the potential for drug development. Hence, exploring the anti-tumor mechanisms of Huaier has become urgently necessary.

In this study, we firstly investigated Huaier inhibited the viabilities of Bel-7404, Bel-7402 and SMMC-7721 cells. Then, our study focused on how Huaier inhibited the proliferation of HCC cells. PCNA is necessary for DNA synthesis in eukaryotic cells, and its expression level represents the proliferative activity of HCC cells [12]. Ki-67 is a kind of nuclear antigen in the process of cell proliferation, and it is highly expressed in various tumor cells, including HCC cells [13]. PCNA and Ki-67 are closely associated with hepatocarcinogenesis, which makes them become ideal bio-markers for HCC cell proliferation. Additionally, CyclinD1 has been recognized as an oncogene, and its overexpression can activate the cyclin dependent kinase 4 (CDK4) during the period of G1, which promotes the cell cycle from G1 to S, as a result, CyclinD1 is able to exert its function of promoting proliferation [14]. Thereby, in this study, we tested whether Huaier was able to inhibit the proliferation of HCC cells by decreasing the levels of PCNA, Ki-67 and CyclinD1. Besides, we also confirmed the apoptosis-induced capacity of Huaier in Bel-7404 and SMMC-7721 cells.

The migration of HCC plays a critical role in the prognosis of tumor, nearly $90 \%$ of HCC-related deaths are due to HCC cell migration [15]. This study also shown Huaier significantly inhibited the migratory abilities of Bel-7404, Bel-7402 and SMMC-7721 cells by regulating EMT. Importantly, the expression levels of E-cadherin and $\mathrm{N}$-cadherin in L-02 cells was almost unaffected after Huaier treatment. 
A

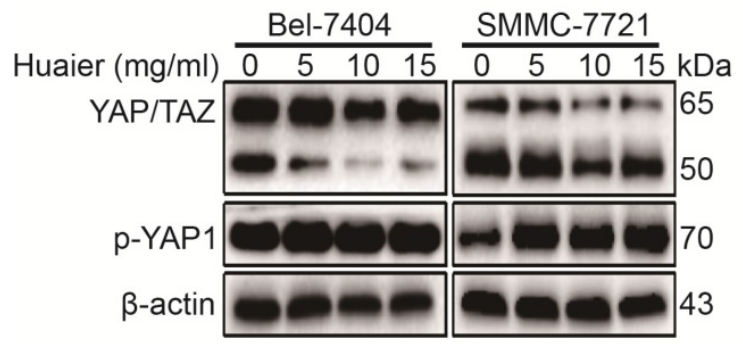

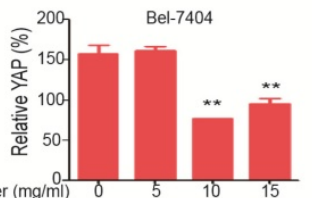
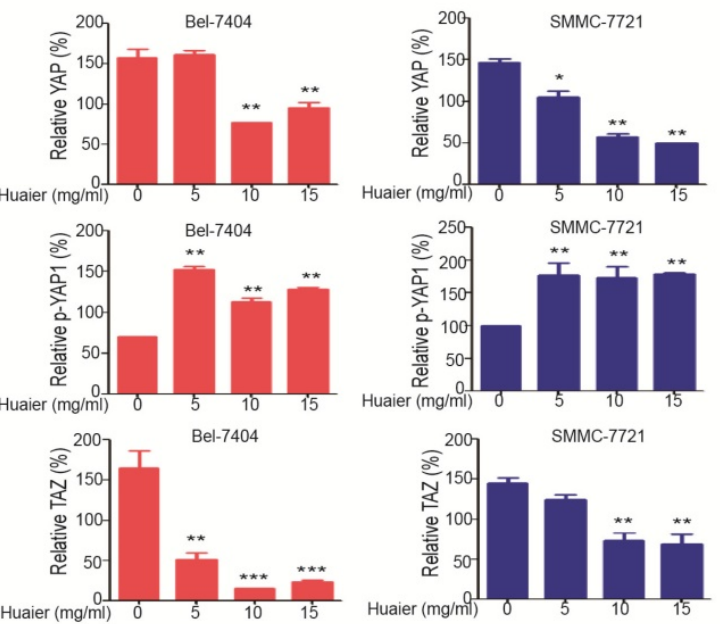

B

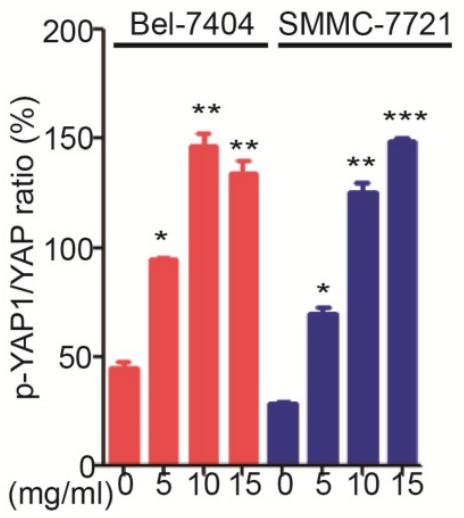

C
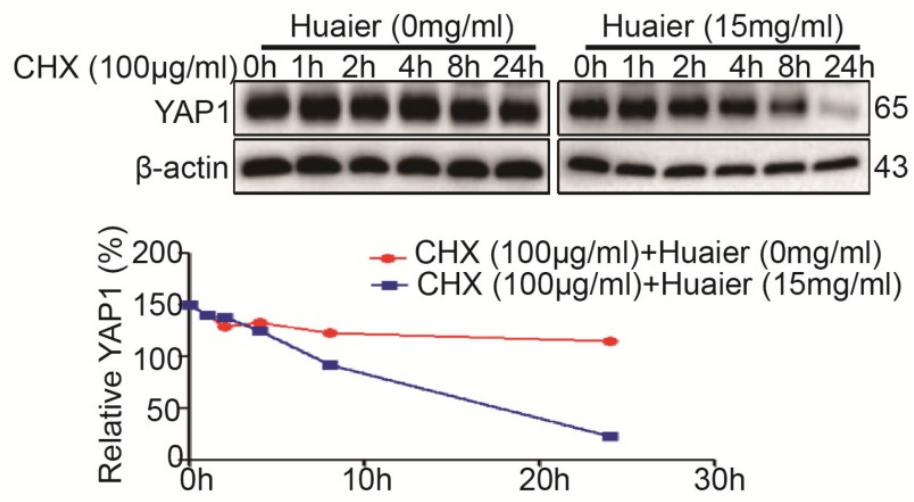

D
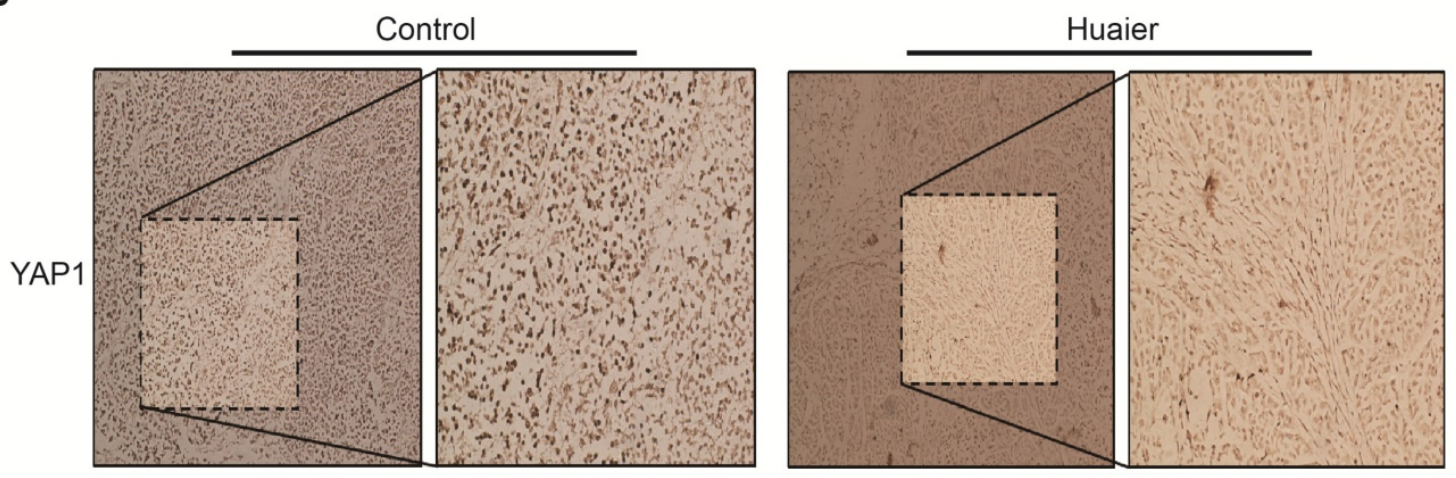

E
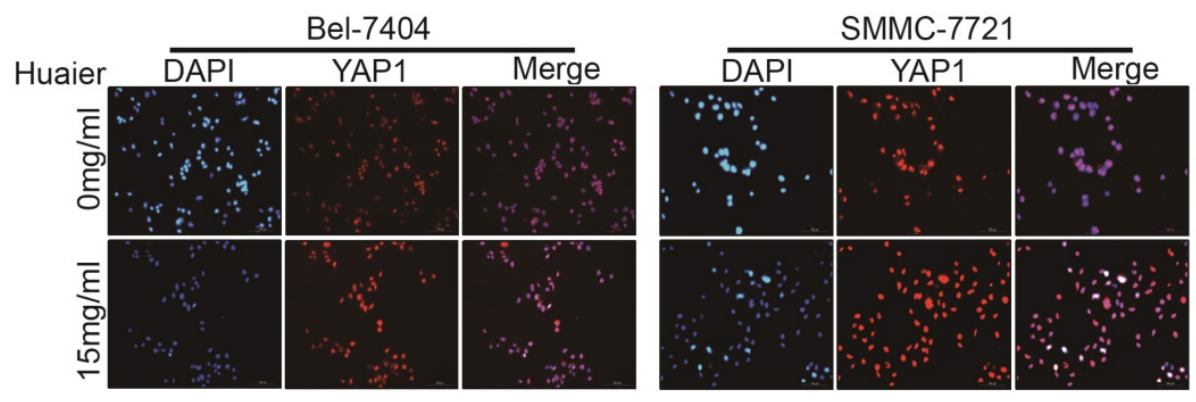

Figure 4. Huaier down-regulated YAPI in HCC cells. A. The protein expressions of YAP/TAZ and p-YAP1 (ser127) in Bel-7404 and SMMC-7721 cells treated with Huaier at the concentrations of $0,5,10$ and $15 \mathrm{mg} / \mathrm{ml}$ for $24 \mathrm{~h}$. Data was represented with GraphPad Prism $5.02 * \mathrm{p}<0.05$, **p<0.01, ***p<0.01 compared with control group. B. The ratio between $\mathrm{P}-\mathrm{Y}$ API (ser 127) and YAP. Data was represented with GraphPad Prism 5.02. ${ }^{*} \mathrm{p}<0.05$, **p<0.01, *** $<<0.01$. C. Effects of CHX $(100 \mu g / \mathrm{ml})$ for $0,1,2,4,8$ and $24 \mathrm{~h}$ on YAP expression in Bel-7404 cells treated with or without Huaier $(15 \mathrm{mg} / \mathrm{ml})$ for $24 \mathrm{~h}$. Data was represented with GraphPad Prism 5.02. D. Effects of Huaier on YAPI in tumors as determined by immunohistochemical analysis. The representative IHC images were taken at a magnification of 20x. E. Immunefluorescence shown Huaier treatment (15mg/ml) for 24h translocated YAPI from nucleus to cytoplasm in Bel-7404 and SMMC-7721 cells. The representative IF images were represented by LASV4.5, $100 \mu \mathrm{m}$. 


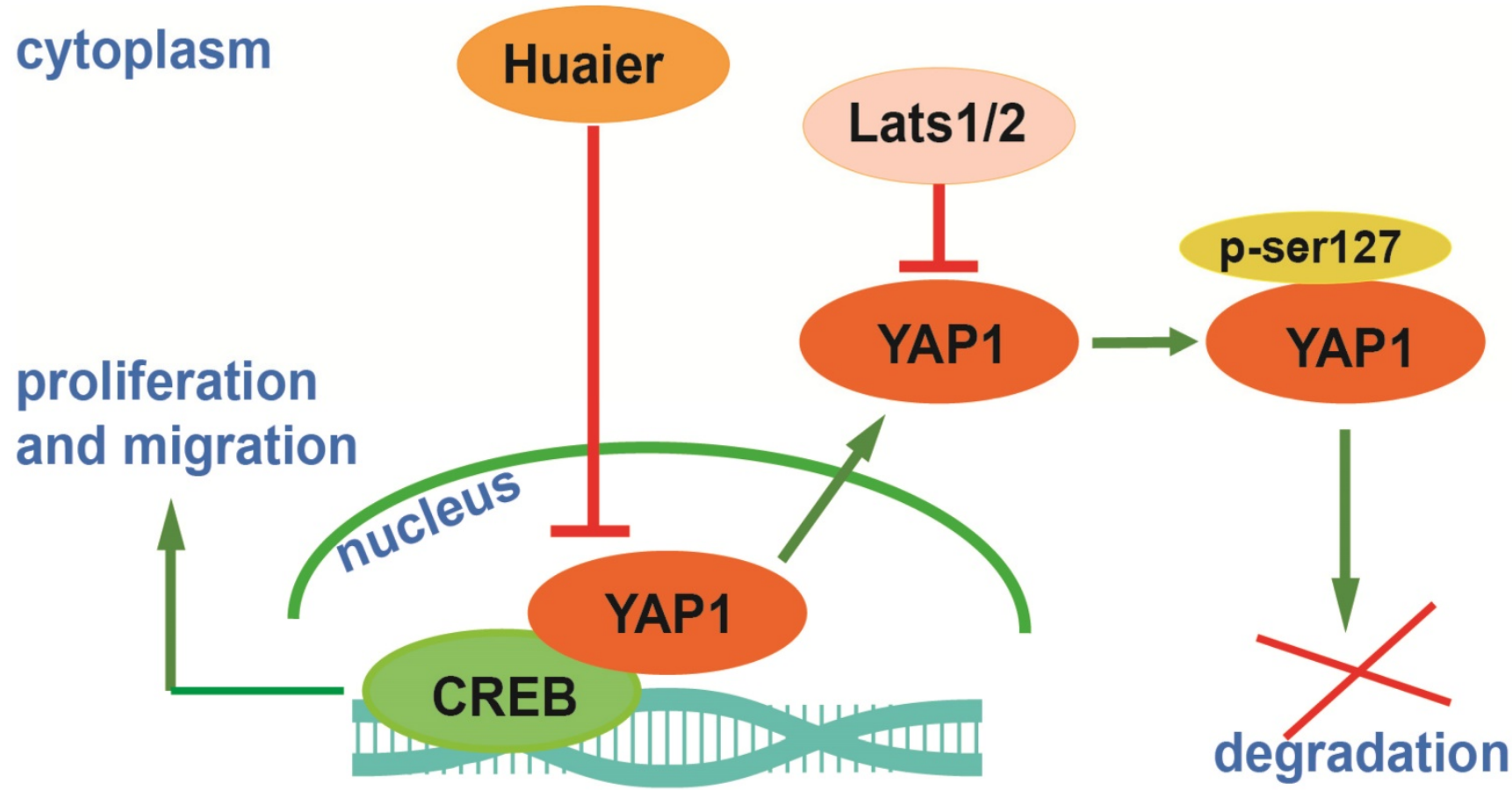

Figure 5. A potential mechanism of Huaier on inhibiting proliferation and migration through decreased YAP1 in HCC cells.

Hepatocarcinogenesis is regulated by numerous factors, we have proved Huaier was able to inhibit Bel-7404, Bel-7402 and SMMC-7721 cells via regulation of PCNA, Ki-67, CyclinD1, Bcl-2, Bax, Caspases, E-cadherin and N-cadherin. Furthermore, we indicated Huaier was able to inhibit Bel-7404 and SMMC-7721 cells by inducing the degradation of YAP1 in vivo and in vitro. Combining with related researches, a molecular network begins to emerge: in the process of hepatocarcinogenesis, overexpression of YAP1 is located in the nucleus, as a result, YAP1 is able to bind to cyclic adenosine monophosphate response element-binding protein (CREB) to promote transcriptional activity of CREB, then YAP1-CREB interaction stimulates the transcription of downstream genes that can promote HCC proliferation and migration [16]. After Huaier treatment, YAP1 exits from the nucleus, and then YAP1 (ser127) is phosphorylated by Lats1/2. Finally, p-YAP1 (ser127) and 14-3-3 are localized in the cytoplasm, and then p-YAP1 is ubiquitinated and degraded $[17,18]$. This mechanism is presented in Figure 5.

Collectively, our study is beneficial for understanding the mechanisms of Huaier on inhibiting proliferation and migration of HCC cells. However, as is known to all, the anti-tumor effects of Huaier are a complicated process, consequently, our study is far from enough. We believe there are still many potential factors that need to be deciphered. Additionally, exploring the interaction between these factors during Huaier treatment is also a great challenge. While revealing the exact mechanisms of Huaier treatment is still a difficult task, as research continues, it is useful for the clinical applications and the development of targeted therapies for HCC.

\section{Acknowledgements}

This study was supported by Natural science foundation of China (Grant No.81472124), Talent introduction project of Shanghai Municipal Hospital of Traditional Chinese Medicine (Grant No.20160501) and Innovation project of Shanghai University of Traditional Chinese Medicine (Grant No. JXDXSCXJH10).

\section{Competing Interests}

The authors have declared that no competing interest exists.

\section{References}

1. Villanueva A, Llovet JM. Targeted therapies for hepatocellular carcinoma. Gastroenterology. 2011; 140: 1410-26.

2. Bao H, Liu P, Jiang K, Zhang X, Xie L, Wang Z, et al. Huaier polysaccharide induces apoptosis in hepatocellular carcinoma cells through p38 MAPK. Oncol Lett. 2016; 12: 1058-66.

3. Zou Y, Xiong H, Xiong H, Lu T, Zhu F, Luo Z, et al. A polysaccharide from mushroom Huaier retards human hepatocellular carcinoma growth, angiogenesis, and metastasis in nude mice. Tumour Biol. 2015; 36: 2929-36.

4. Zheng J, Li C, Wu X, Liu M, Sun X, Yang Y, et al. Astrocyte elevated gene-1 (AEG-1) shRNA sensitizes Huaier polysaccharide (HP)-induced anti-metastatic potency via inactivating downstream P13K/Akt pathway as well as augmenting cell-mediated immune response. Tumour Biol. 2014; 35: 4219-24.

5. Cai WY, Lin LY, Hao H, Zhang SM, Ma F, Hong XX, et al. Yes-associated protein/TEA domain family member and hepatocyte nuclear factor 4-alpha (HNF4alpha) repress reciprocally to regulate hepatocarcinogenesis in rats and mice. Hepatology. 2017; 65: 1206-21. 
6. Rongvaux A, Jackson R, Harman CC, Li T, West AP, de Zoete MR, et al. Apoptotic caspases prevent the induction of type I interferons by mitochondrial DNA. Cell. 2014; 159: 1563-77.

7. White MJ, McArthur K, Metcalf D, Lane RM, Cambier JC, Herold MI, et al Apoptotic caspases suppress mtDNA-induced STING-mediated type I IFN production. Cell. 2014; 159: 1549-62.

8. Huo $\mathrm{Q}$, Ge $\mathrm{C}$, Tian $\mathrm{H}$, Sun J, Cui $\mathrm{M}$, Li $\mathrm{H}$, et al. Dysfunction of IKZF1/MYC/MDIG axis contributes to liver cancer progression through regulating H3K9me3/p21 activity. Cell Death Dis. 2017; 8: e2766.

9. He C, Zhou Z, Jiang H, Yin Z, Meng S, Zhang J, et al. Epithelial-Mesenchymal Transition is Superior to Vessels-Encapsulate Tumor Cluster in Promoting Metastasis of Hepatocellular Carcinoma: a Morphological Evidence. J Cancer. 2017; 8: 39-47.

10. Kim W, Khan SK, Gvozdenovic-Jeremic J, Kim Y, Dahlman J, Kim H, et al. Hippo signaling interactions with Wnt/beta-catenin and Notch signaling repress liver tumorigenesis. J Clin Invest. 2017; 127: 137-52.

11. Song X, Li Y, Zhang H, Yang Q. The anticancer effect of Huaier (Review). Oncol Rep. 2015; 34: 12-21.

12. Strzalka W, Ziemienowicz A. Proliferating cell nuclear antigen (PCNA): a key factor in DNA replication and cell cycle regulation. Ann Bot. 2011; 107: 1127-40.

13. Schmilovitz-Weiss H, Tobar A, Halpern M, Levy I, Shabtai E, Ben-Ari Z. Tissue expression of squamous cellular carcinoma antigen and Ki67 in hepatocellular carcinoma-correlation with prognosis: a historical prospective study. Diagn Pathol. 2011; 6: 121

14. Qu K, Wang Z, Fan H, Li J, Liu J, Li P, et al. MCM7 promotes cancer progression through cyclin D1-dependent signaling and serves as a prognostic marker for patients with hepatocellular carcinoma. Cell Death Dis. 2017; 8: e2603.

15. Zhai W, Lim TK, Zhang T, Phang ST, Tiang Z, Guan P, et al. The spatial organization of intra-tumour heterogeneity and evolutionary trajectories of metastases in hepatocellular carcinoma. Nat Commun. 2017; 8: 4565.

16. Wang J, Ma L, Weng W, Qiao Y, Zhang Y, He J, et al. Mutual interaction between YAP and CREB promotes tumorigenesis in liver cancer. Hepatology. 2013; 58: 1011-20.

17. Lee DH, Park JO, Kim TS, Kim SK, Kim TH, Kim MC, et al. LATS-YAP/TAZ controls lineage specification by regulating TGFbeta signaling and Hnf4alpha expression during liver development. Nat Commun. 2016; 7: 11961.

18. Oh H, Irvine KD. Yorkie: the final destination of Hippo signaling. Trends Cell Biol. 2010; 20: 410-7. 